\title{
Pulmonary Hyalinising Granuloma A report of two cases
}

Ameen Kamona, ${ }_{1}$ Fatma Al Lawati, ${ }^{2}$ Atheel Kamona, ${ }^{3}$ Nasser Al Busaidi, ${ }^{4}$ Yaqoob Al Mahrooqi, ${ }^{4}$ Saqar Al-Tai, Nabil Al Lawati, ${ }^{4}$ Rashid S. Al-Umairi ${ }^{3}$

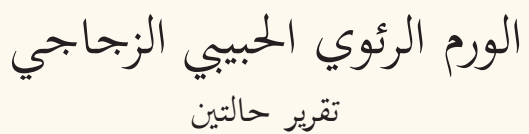

امين كمونة، فاطمة اللواتية، اثيل كمونة، ناصر البوسعيدي، يعقوب المحروقي، صقر الطائي، نبيل اللواتي، راشد بن سيف العميري

ABSTRACT: Pulmonary hyalinising granuloma (PHG) is a rare fibrosclerosing inflammatory lung condition of unknown aetiology. It is characterised by solitary or multiple pulmonary nodules that are usually found incidentally while imaging the chest for other reasons. We report two cases of histologically proven PHG diagnosed at the Royal Hospital, Muscat, Oman. The first case was a 71-year-old male patient who presented in 2010 with a dry cough, weight loss and bilateral pulmonary nodules. The second case was a 58-year-old male patient who presented in 2012 and was found to have incidental bilateral pulmonary nodules on chest X-ray. Both patients were started on prednisolone and on follow-up the PHG nodules remained stable. Although there is no definitive treatment, PHG generally has an excellent prognosis.

Keywords: Granuloma; X-Ray Computed Tomography; Multiple Pulmonary Nodules; Lung; Case Report; Oman.

الملخص: الورم الرئوي الحبيبي الزجاجي هو نوع نادر من التهابات الرئة المتصفة بـالتليف والتحجر والذي لودئ لا يعرف مسببه حتى الآن.

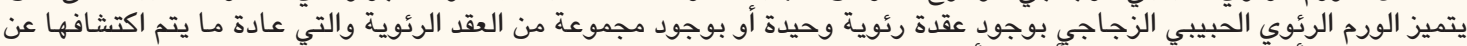

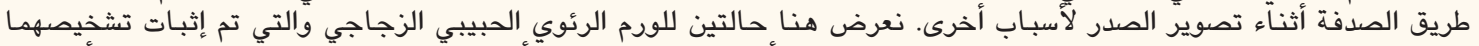

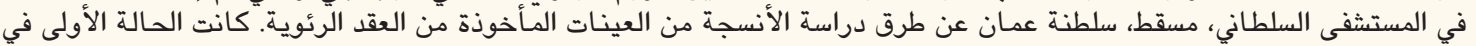

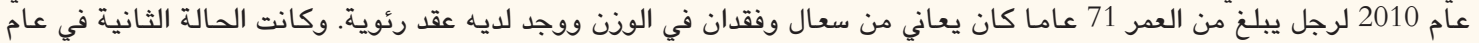

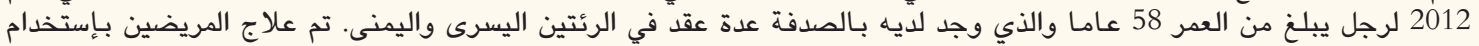

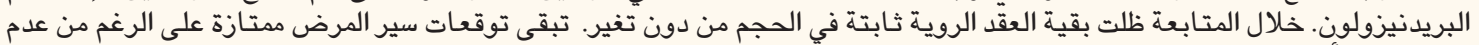

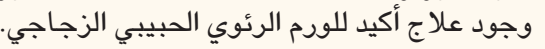

الكلمات المفتاحية: ورم حبيبي؛ أشعة سينية مقطعية؛ عقيدات رئوية متعددة؛ رئة؛ تقرير حالة؛ عمان.

$\mathrm{P}$ ULMONARY HYALINISING GRANULOMA (PHG) is a rare fibrosclerosing inflammatory lung disease which was first reported by Benfield et al. in 1962 and then characterised by Engleman et al. in $1977.1,2$

Patients with PHG might have non-specific mild symptoms or be asymptomatic. The presence of PHG is usually discovered incidentally by chest X-ray or computed tomography (CT) scan performed for other reasons. ${ }^{3}$ PHG is characterised by either unilateral or bilateral multiple pulmonary nodules which consist of extracellular, eosinophilic hyaline lamellae, surrounded by a collection of plasma cells, lymphocytes and histiocytes. ${ }^{2-5}$ Although an exaggerated immune response to the antigenic stimuli by infection or autoimmune processes has been proposed as an aetiology, the exact pathophysiology of PHG remains unknown. ${ }^{3}$

On imaging, PHG presents as solitary or multiple pulmonary nodules that can mimic other more common pulmonary disorders, such as neoplastic and granulomatous conditions. ${ }^{3}$ To the best of the authors' knowledge, these are the first cases of PHG reported in Oman.

\section{Case One}

A 71-year-old male patient was referred to the Royal Hospital, Muscat, Oman, in 2010 for the further work-up of lung lesions found on a chest CT scan. He had diabetes and hypertension and complained of a six-month history of dry cough associated with weight loss. There was no history of smoking, haemoptysis, anorexia or fever. On physical examination, there were bilateral palpable supraclavicular lymph nodes, but an otherwise unremarkable systemic examination. Laboratory tests were normal, including complete blood count, renal function test, liver function test, rheumatoid factor and antinuclear antibody. Chest X-ray showed bilateral pulmonary nodules and further evaluation 


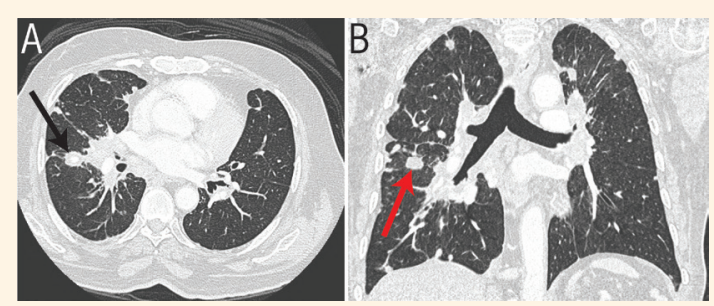

Figure 1: High resolution computed tomography scans of the chest of a 71-year-old male patient (case one) in (A) axial and (B) coronal views showing bilateral pulmonary nodules (black and red arrows) and central calcification in the right upper lobe nodule (red arrow).

with a chest CT scan revealed bilateral pulmonary nodules and masses associated with nodular interlobular septal thickening, along with extensive bilateral hilar and mediastinal lymphadenopathy. Some pulmonary nodules showed central calcification [Figure 1]. There was a small right-sided pleural effusion. Findings from the chest CT scan were suggestive of a metastatic process from an unknown primary source. F-18-fluordeoxyglucose positron emission tomography/CT (FDGPET/CT) was performed; however, no extrathoracic lesion could be seen. The decision was made to obtain a tissue sample from the lung nodules.

Bronchoscopy was performed and bronchoalveolar lavage (BAL) was tested. Findings were negative for malignant cells and acid-fast bacilli (AFB). The tuberculosis (TB) culture from bronchial lavage was also negative. Subsequently, CT-guided biopsy of the pulmonary nodule was performed. Microscopic examination of the obtained tissue sample using haematoxylin and eosin stain showed a thick relatively acellular eosinophilic collagen bundle with lamellar arrangement that formed a storiform pattern [Figure 2]. The Congo red stain for amyloid was negative. Histopathological findings were consistent with PHG.

The patient was started on prednisolone $30 \mathrm{mg}$ for two months and then $5 \mathrm{mg}$ for another ten months. The PHG nodules remained stable on follow-up; however, the patient developed prostate cancer after three years following the diagnosis of PHG.

\section{Case Two}

A 58-year-old male patient with a known history of paraparesis due to myelopathy since 2009 presented to the neurology clinic at the Royal Hospital, in 2012 with a history of painless haematuria, significant weight loss and generalised abdominal pain. The patient had no history of smoking. Physical examination was normal except for rhonchi on the left lung and stable neurological deficits. Urine analysis was positive for blood, leukocytes and protein. Other laboratory tests were normal, including complete blood count and renal and liver function tests.

Abdominal ultrasound showed diffuse bladder wall thickening and trabeculation with a moderate right hydroureteronephrosis; no renal calculi were detected. The patient was treated as having a urinary tract infection; however, his chest X-ray showed bilateral multiple pulmonary nodules and masses [Figure 3]. CT scans of the abdomen, chest and pelvis were performed for further work-up and showed multiple pulmonary nodules and masses, some of which were calcified [Figure 4]. There were also multiple enlarged mediastinal lymph nodes and some had evidence of calcification with no identifiable primary source. Based on the CT findings, radiological differential diagnoses included lymphoma and metastasis from an unknown primary and histopathological correlation was recommended. Bone scan showed no evidence of bone metastasis. Both the AFB test and TB culture were negative.

Bronchoscopy was performed and BAL was negative for malignant cells as well as for AFB. The TB culture from the bronchial lavage was also negative. Pulmonary nodules were further evaluated with a CTguided lung biopsy and the histopathological report was inconclusive. Subsequently, a right thoracoscopic
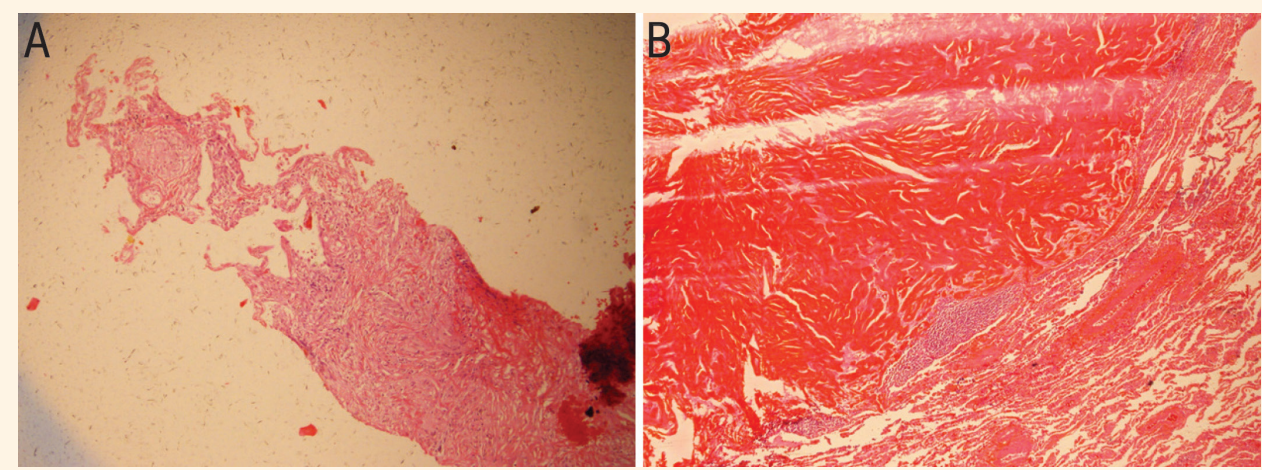

Figure 2: Haematoxylin and eosin stains of a pulmonary hyalinising granuloma nodule tissue sample obtained from a 71-year-old male patient (case one). A: Lung core biopsy at x 5 magnification showing fascicles of collagen. B: Lung wedge biopsy at $\mathrm{x} 2$ magnification showing keloid-like collagen surrounded by a rim of lymphocytes. 


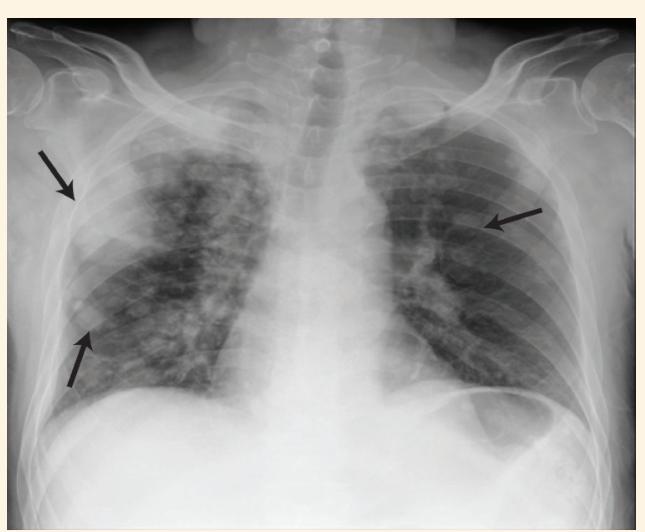

Figure 3: Coronal chest X-ray of a 58-year-old male patient (case two) showing bilateral pulmonary nodules and masses (black arrows), predominantly in the right lung.

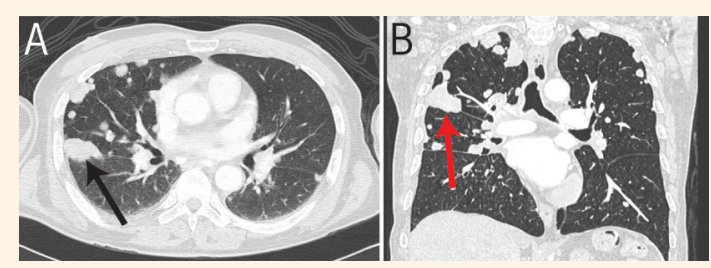

Figure 4: High resolution computed tomography scans of the chest of a 58-year-old male patient (case two) in (A) axial and (B) coronal views showing bilateral pulmonary nodules (black and red arrows) and central calcification in the right upper lobe nodule (red arrow).

lung biopsy and nodular excision were performed. Histopathological findings of the excised tissue were consistent with PHG.

The patient was counselled about the benign nature of the diagnosis and treated with prednisolone, initially $30 \mathrm{mg}$ for three months and then gradually decreased over another three months. The patient was followed-up for five years and the pulmonary nodules remained stable. However, the patient developed Hodgkin's lymphoma five years following the diagnosis of PHG.

\section{Discussion}

PHG is a rare benign pulmonary disorder that is characterised by single or multiple pulmonary nodules. It is more predominant in males, with a mean age of 44.6 years (range: $15-83$ years) at presentation. ${ }^{3}$ Patients with PHG can present with mild and non-specific symptoms such as cough, fever, fatigue, dyspnoea and pleuritic chest pain. However, up to $25 \%$ of patients might be completely asymptomatic, with the pulmonary lesions detected incidentally while imaging the chest for other reasons. In the current cases, the first patient had a history of cough for six months associated with weight loss, while the second patient had pulmonary nodules that were detected incidentally while the patient was being evaluated for abdominal complaints.

Although the cause of PHG is unknown, it has been associated with infection and autoimmune and neoplastic disorders. ${ }^{3,4,6-12}$ Lhote et al. reported a series of five PHG cases and reviewed 135 cases in the literature; they found that 19 cases were associated with infectious causes, of which TB was the most common infection. In the same study, 17 cases were associated with auto-immune disease and an association with mediastinal and retroperitoneal fibrosis was found in 19 and 12 cases, respectively. ${ }^{3}$ Therefore, it has been proposed that PHG might be caused by an abnormal immune response to all of the above conditions. Chapman et al. reported a case of PHG and found elevated serum immunoglobulin (Ig)G4 and tissue IgG4-postive plasma cell in the PHG and concluded that PHG is part of IgG4 related-sclerosing disease. ${ }^{12}$ In the current cases, neither of the patients were tested for IgG4 level.

In Lhote et al's study, PHG was found to be associated with solid tumours in six cases, including breast Paget's disease, lung adenocarcinoma, meningioma, anaplastic astrocytoma, thyroid carcinoma and basocellular carcinoma. ${ }^{3}$ Ren et al. reported an association between PHG and lymphoma. ${ }^{13}$ The current patients had developed two different malignant tumours on follow-up: the first patient developed prostate cancer, while the second developed Hodgkin's lymphoma. This suggests a possible association between PHG and the future development of malignancies, which needs to be further explored.

On chest X-ray and CT, PHG is characterised by solitary or, more often, multiple randomly distributed, unilateral or bilateral pulmonary nodules and/or masses without preferential distribution. The PHG nodules are usually well-defined and homogeneous, ranging in size from several millimetres to $5 \mathrm{~cm}$ in diameter, with an average diameter of $2 \mathrm{~cm}$. They can calcify but rarely cavitate. Up to $60 \%$ of PHG lesions show increased metabolic activity using FDG-PET/CT.3-12,14-16

Radiological findings in PHG can mimic other nodular pulmonary disorders such as neoplastic and granulomatous processes; hence, radiological differential diagnosis should include sarcoidosis, rheumatoid nodules, Wegener's granulomatosis, TB and amyloidosis, as well as primary or metastatic tumours of the lung. $3,9,10,12,15$ Therefore, histopathological examination is required to diagnose PHG and differentiate it from other nodular pulmonary disorders.

In the present two cases, the radiological findings of both patients are similar to those reported in the literature, with bilateral randomly distributed pulmonary nodules, some of which showed calcification. Both 
patients had mediastinal lymphadenopathy, with some lymph nodes in the second patient showing central calcification.

Microscopically, PHG lesions are composed of a concentric dense network of hyalinised collagen that is centred in the perivascular lymphoplasmacytic infiltrate located in the centre of PHG lesions. ${ }^{3,6,8-10,12,16}$

The prognosis for patients with PHG is generally excellent. On follow-up, PHG shows a variable course with some nodules changing size and some remaining stable. Multiple lesions usually show a more rapid growth rate compared to single lesions.,12

There is no definitive treatment for PHG. Although radiological and symptomatic improvement or even successful resolution of the nodules with the administration of glucocorticoids has been reported, the effect of corticosteroids remains unclear., ${ }^{3,5}$ Surgical resection in patients with a solitary PHG nodule has been reported as a cure. ${ }^{3,4,12}$ The current two patients received oral prednisolone; however, the nodules remained stable in size on follow-up chest CT scan.

\section{Conclusion}

PHG is a rare fibrosclerosing inflammatory pulmonary disease that can mimic other nodular pulmonary disorders such as neoplastic and granulomatous processes; therefore, it should be included in the differential diagnosis of pulmonary nodules or masses. Few reported cases of PGH in the literature developed extra-pulmonary malignancy on follow-up, however, this was the case for both current patients. There is a possible association between PHG and the future development of malignancy, which needs to be further explored and should warrant close follow-up of patients with proven PHG.

\section{CONFLICT OF INTEREST}

The authors declare no conflicts of interest.

\section{FUNDING}

No funding was received for this study.

\section{References}

1. Benfield JR, Harrison RW, Moulder PV, Lyon ES, Graff PW. Bilateral nodular pulmonary granulomas and retroperitoneal fibrosis. Simulated metastatic malignant neoplasm and spontaneous remission of ureteral obstruction. JAMA 1962; 182:579-81. https://doi.org/10.1001/jama.1962.03050440071021e.
2. Engleman P, Liebow AA, Gmelich J, Friedman PJ. Pulmonary hyalinizing granuloma. Am Rev Respir Dis 1977; 115:997-1008.

3. Lhote R, Haroche J, Duron L, Girard N, Lafourcade MP, Martin M, et al. Pulmonary hyalinizing granuloma: A multicenter study of 5 new cases and review of the 135 cases of the literature. Immunol Res 2017; 65:375-85. https://doi.org/10.1007/s12026-0 16-8852-4.

4. Uçvet A1, Tözüm H, Gürsoy S, Gülle AA, Yaldiz S, Aydoğdu Dinç Z. [Pulmonary hyalinizing granuloma mimicking pulmonary carcinoma]. Tuberk Toraks 2006; 54:71-4.

5. Shinohara T, Kaneko T, Miyazawa N, Nakatani Y, Nishiyama H, Shoji A, et al. Pulmonary hyalinizing granuloma with laryngeal and subcutaneous involvement: Report of a case successfully treated with glucocorticoids. Intern Med 2004; 43:69-73.

6. Ussavarungsi K, Khoor A, Jolles HI, Mira-Avendano I. A 40-yearold woman with multiple pulmonary nodules. Pulmonary hyalinizing granuloma. Chest 2014; 146:e198-203. https://doi.org/10.1 378/chest.14-0796.

7. Lien CT, Yang CJ, Yang SF, Chou SH, Huang MS. Pulmonary hyalinizing granuloma mimicking multiple lung metastases: Report of fluorodeoxyglucose positron emission findings. J Thorac Imaging 2010; 25:W36-9. https://doi.org/10.1097/RTI.0b01 3e3181a4bb41.

8. Kaneta T, Saito A, Akiyama T, Takahashi Y, Takahashi R, Kudou K. [A case of pulmonary hyalinizing granuloma with high-grade uptake on FDG-PET mimicking metastatic lung cancer]. Nihon Kokyuki Gakkai Zasshi 2009; 47:953-9.

9. Düzgün N, Kurtipek E, Esme H, Eren Karanis Mİ, Tolu İ. Pulmonary hyalinizing granuloma mimicking metastatic lung cancer. Case Rep Pulmonol 2015; 2015:610417. https://doi.org/10.1155/2 015/610417.

10. Colen RR, Nagle JA, Wittram C. Radiologic-pathologic conference of the Massachusetts General Hospital. Pulmonary hyalinizing granuloma. AJR Am J Roentgenol 2007; 188:W15-16. https://doi.org/10.2214/ajr.06.0380.

11. Coleman C, Nassar A, McComb B. Pulmonary hyalinizing granuloma associated with idiopathic thrombocytopenic purpura. J Clin Imaging Sci 2014; 4:8. https://doi.org/10.4103/21567514.127835.

12. Chapman EM, Gown A, Mazziotta R, Churg A. Pulmonary hyalinizing granuloma with associated elevation in serum and tissue IgG4 occurring in a patient with a history of sarcoidosis. Am J Surg Pathol 2012; 36:774-8. https://doi.org/10.1097/PAS.0 b013e318248713d.

13. Ren Y, Raitz EN, Lee KR, Pingleton SK, Tawfik O. Pulmonary small lymphocytic lymphoma (mucosa-associated lymphoid tissue type) associated with pulmonary hyalinizing granuloma. Chest 2001; 120:1027-30. https://doi.org/10.1378/chest.120.3.1027.

14. Satti MB, Batouk AA, Abdelaziz MM, Ahmad MF, Abdelaal MA. Pulmonary hyalinizing granuloma. Bilateral pulmonary nodules associated with chronic idiopathic thrombocytopenic purpura. Saudi Med J 2005; 26:1459-63.

15. Lvovsky D, Rosman J, Iliescu ME. Pulmonary hyalinizing granuloma (PHG) presenting as bilateral dense apical masses. Chest 2004; 126:950S. https://doi.org/10.1378/chest.126.4_Mee tingAbstracts.950S

16. Atagi S, Sakatani M, Akira M, Yamamoto S, Ueda E. Pulmonary hyalinizing granuloma with Castleman's disease. Intern Med 1994; 33:689-91. https://doi.org/10.2169/internalmedici ne.33.689. 